\title{
Sensitive immunoassay of cardiac troponin I using an optimized microelectrode array in a novel integrated microfluidic electrochemical device
}

\author{
Yang $\mathrm{Li}^{1}$, Shaohua Zuo ${ }^{1}$, Laiqian Ding ${ }^{1}$, Pengpeng $\mathrm{Xu}^{1}$, Kun Wang ${ }^{1}$, Yuanchang $\mathrm{Liu}^{2}$, Jingmin $\mathrm{Li}^{1 *}$, \\ Chong $\mathrm{Liu}^{1,3^{*}}$ \\ ${ }^{1}$ Key Laboratory for Micro/Nano Technology and System of Liaoning Province, Dalian University of Technology, Dalian, China \\ ${ }^{2}$ Department of Mechanical Engineering, University College London, Torrington Place, London WC1E 7JE, UK \\ ${ }^{3}$ Key Laboratory for Precision and Non-traditional Machining Technology of Ministry of Education, Dalian University of \\ Technology, Dalian, China \\ *Corresponding author. \\ E-mail address: chong1@dlut.edu.cn (Chong Liu), jingminl@dlut.edu.cn (Jingmin Li). \\ Post address: Key Laboratory for Micro/Nano Technology, Dalian University of Technology, No.2 Linggong Road, Ganjingzi \\ District, Dalian City, Liaoning Province, P.R.C.
}

Key words: Immunoassay, $\mu$ FED, SAMs, Shielding Effect

\begin{abstract}
A sensitive and portable microfluidic electrochemical array device ( $\mu$ FED) was developed for the immunoassay of human cardiac troponin I (cTnI) in trace amount, which is an attractive biomarker for acute myocardial infarction (AMI). The classical "sandwich-method" was adopted for the immunoassay. The capture antibody was immobilized by self-assembled monolayers (SAMs) technique, and its process is reorganized to be compatible with bonding process. The detection antibody was labeled with alkaline phosphatase (AP) for the signal amplification. The performance of the $\mu$ FED was improved by eliminating the shielding effect of the microelectrode array (MEA) integrated in $\mu$ FED. The effects of the interstice and the width of the MEA on the response peak current were analyzed and simulated. The concentration gradient, about $3 \%$ of the gradient at the surface, could be considered as the criterion for estimation of the optimal interstice between electrodes, and its effectiveness was proved. A stable and miniaturized reference electrode was integrated in the $\mu \mathrm{FED}$, and its potential deviation was less than 5 $\mathrm{mV}$ in $15 \mathrm{~min}$. These efforts resulted the amplification of the immunoassay performance of the $\mu \mathrm{FED}$. A low limit of detection about $5 \mathrm{pg} / \mathrm{ml}$ was obtained in serum sample, and the response current was proportional to the logarithm of concentration from $50 \mathrm{pg} / \mathrm{ml}$ to $1 \mu \mathrm{g} / \mathrm{ml}$. The immunoassay process could be accomplished in $15 \mathrm{~min}$. The $\mu \mathrm{FED}$ was qualified and promising for the point-of-care immunoassay of cTnI.
\end{abstract}

\section{Introduction}

Acute myocardial infarction (AMI) is one of the most threatening cardiovascular diseases and a major cause of morbidity and mortality, and electrocardiogram (ECG) is the mainstay initial screening test for the diagnosis of AMI, but only less than one-third of potential patients admitted to emergency departments exhibit definitive ECG evidence of AMI, thus the measurements of biomarkers are always required in the further diagnosis of the remaining patients [1-4]. When the damage or death of heart muscle happens, which could result in AMI, cTnI is released into blood rapidly, leading to a significant concentration elevation within 3-4 hours, and such an elevation will remain for 4-10 days, thus cTnI has been considered as a promising biomarker for AMI [5]. The concentration level of cTnI is normally lower than $0.1 \mathrm{ng} / \mathrm{mL}$, and a level between 0.1 and $2 \mathrm{ng} / \mathrm{mL}$ indicates a diagnosis of unstable angina and other 
heart disorder, whereas levels greater than $2 \mathrm{ng} / \mathrm{mL}$ indicate an increasing risk for future serious heart events $[2,6,7]$. But, a timely, accurate, convenient and "near patients" diagnosis technique is still an unmet clinical need for daily diagnosis of susceptible populations.

The microfluidic electrochemical array device ( $\mu \mathrm{FED}$ ) is a promising platform $[8,9]$, and has received a major share of attention in the development for disease diagnosis[10,11] using nanoliter to microliter scale volumes of sample [12,13]. Among the electroanalytical approaches explored to measure biomarkers of diseases, amperometric detection is more promising to be integrated in $\mu$ FED for on-site application [14]. Andrea et al. described an electrochemical aptamer-based sensor for the detection of clinical tryptophan levels in $10 \mathrm{~min}$, and the sensor could measure the entire clinical range (from 0.1 to $10 \mathrm{mM}$ ) [15]. Carvajal et al. presented a disposable device based on inkjet-printed electrochemical sensor array for immunosensing of clinically relevant HER-2 in serum, and a low detection limit of $12 \mathrm{pg} / \mathrm{ml}$ could be achieved in $15 \mathrm{~min}$ [16]. Although $\mu \mathrm{FED}$ has been widely reported, its response signal of the micro electrode array (MEA) in $\mu \mathrm{FED}$ was always improved and amplified by the modification of nano structure or functional materials with high electrical conductivity and surface electroactive sites [7,17], but limited studies focusing on the effective optimization methods for the structure parameters of MEA, especially the interstice between adjacent microelectrodes. The performance of unoptimized MEAs would be compromised by the shielding effect, because of the overlaps of diffusion fields between adjacent electrodes. It is evident that the efficiency of mass transfer and the concentration gradient in the overlapping diffusion field will be reduced, which will result in a significant reduction of response current. Even the enlargement of the interstice can eliminate the shielding effect, but an excessive and aimless increase of the interstice goes against both the miniaturization of the MEA and the demand for small volume sample. The key to optimize the MEA lies on finding the thickness of diffusion field. The optimal interstice should be twice of the thickness so that the diffusion fields should be tangential exactly without any shielding effect. Additionally, the miniaturized and integratable reference electrode in $\mu \mathrm{FED}$ is always neglected. The reference electrode was always immersed in the analyte sample[18,19]. In practical application, the incomplete reference electrode cannot offer a stable and reliable reference potential for the electrochemical measure system without certain supporting electrolyte in a certain concentration. Consequently, the potential applied on the MEA may drift, and the interference reactant may react at the surface of the working electrode, even cause the corrosion of the electrode (as shown in Fig.S1). Those may limit the development and application of $\mu$ FED.

In this study, we designed a sensitive and portable $\mu \mathrm{FED}$ for the immunoassay of $\mathrm{cTnI}$ in serum and demonstrated the effectiveness of the device. To make the maximum advantage of MEA in the $\mu$ FED and eliminate the "shielding effect", we attempted to deduce a criterion for the optimal interstice of MEA with groups of experiment and a simulation model about the diffusion distribution, and as a basis for the design of the MEA for the immunoassay of cTnI. We also integrated a miniaturized $\mathrm{Ag} / \mathrm{AgCl}$ reference electrode in the $\mu \mathrm{FED}$ to improve the stability of the electrochemical measurement. A supporting electrolyte chamber for reference electrode was designed to maintain the concentration of electrolyte so that the error of reference potential is acceptable. The "sandwich-method" was chosen for the immunoassay. The capture antibody is immobilized by self-assembled monolayers (SAMs) technique. The SAMs process is reorganized to be compatible with bonding process. The alkaline phosphatase (AP) was employed as the amplification label for the trace amount of cTnI, the electrochemical response current of enzymatic products is proportional to cTnI. These efforts improved the performance of the $\mu \mathrm{FED}$, and the immunoassay of $\mathrm{cTnI}$ in serum could be completed in 15 minutes. The response signal is linear to the logarithmic concentration of cTnI over the range from $50 \mathrm{pg} / \mathrm{ml}$ and $1 \mu \mathrm{g} / \mathrm{ml}$, and the limit 
of detection is about $5 \mathrm{pg} / \mathrm{ml}(\mathrm{S} / \mathrm{N}=3)$. The performance of the proposed $\mu$ FED could afford the demands of the risk evaluation of AMI.

\section{Materials and Methods}

Materials and Instrumentations All the information about the chemical reagents and instruments are available in the Supplementary Material.

Fabrication of $\boldsymbol{\mu F E D}$ The device is composed with a strip MEA based electrochemical system and cover plate with a microfluidic network, as shown in Fig.S2. The immunoassay could be accomplished with $0.75 \mu \mathrm{L}$ serum sample. The detail fabrication process is described in the Supplementary Material.

The optimization method of the interstice in MEA The shielding effect, which mainly hinders the performance of MEA, is generated by the overlap of diffusion fields of adjacent electrodes. As the overlaps normally occur at the efficient quarter-cylindrical diffusion fields corresponding to the edges of the strip electrodes, the efficiency of mass transfer and the concentration gradient can be reduced in the overlapping area resulting a significant drop in the response current. The performance of strip MEA with insufficient interstice will be compromised and be similar to the behavior of macroscopic plate electrode consequently. An increase of the interstice between electrodes $\left(W_{i}\right)$ can reduce the overlap of diffusion field and eliminate the shielding effect, but an excessive large $W_{i}$ goes against both the miniaturization of the MEA and the demand for small volume sample. Some design guidelines for MEA in long time electrolyzation are reported, and $W_{i}$ of MEA is recommended as at least 6 times of the width of single electrode $\left(W_{e}\right)$ to avoid the shielding effect [20,21]. But in specific measurement method with certain parameters, the conclusion is not accurate enough, and the size of the $W_{i}$ can be further reduced.

In order to find the optimal $W_{i}$ for the MEA in the $\mu$ FEDs, groups of $\mu$ FEDs were tested in a solution of $0.5 \mathrm{mM} \mathrm{K}_{4}\left[\mathrm{Fe}(\mathrm{CN})_{6}\right]$ and $0.1 \mathrm{M} \mathrm{KCl}$ by cyclic voltammetry $(\mathrm{CV})$ method at a scan rate of $100 \mathrm{mV} / \mathrm{s}$. In the first group of $\mu \mathrm{FEDs}$, the $W_{e}$ of MEAs is $10 \mu \mathrm{m}$ and $W_{i}$ varies from $10 \mu \mathrm{m}$ to $60 \mu \mathrm{m}$, which is 6 times of the width. The variation of the oxidation peak response current with the increase of interstice is analyzed and fitted, and the optimal $W_{i}$ shall be found at the inflection point. To verify whether the change of $W_{e}$ of the MEA affects the value of optimal $W_{i}$, another group of $\mu \mathrm{FEDs}$, whose $W_{e}$ is $20 \mu \mathrm{m}$, were also tested in the same solution with the same parameters.

We also attempted to find a criterion for the optimal interstice of MEA. For the electrochemical reaction controlled by the diffusion process, the distribution of concentration and concentration gradient of the reactant near the electrode surface are the key indicators, not only for the diffusion speed but also the influence factors of the electrochemical response signal. The factors were analyzed with a 2D simulation model about the cross section of single electrode, which was configurated with the Electroanalysis (elan) interface of COMSOL Multiphysics (Version 4.3a, Burlington, MA). The correlation between the response current and these factors was analyzed. The factors can be potentially considered as the criterion were screened out. Their validity for finding the optimal $W_{i}$ was further verified with groups of repeated tests and simulation with different scan rate.

Functional Modification of the Reaction Channel The capture antibody (the M18 monoclonal anticTnI antibody) is employed as the specific recognition biomolecule in the immunosensing process, and it should be immobilized on certain surface before use. The immobilization is realized by self-assembled 
monolayers (SAMs) technique, which is an extensive and convenient platform, and the immobilized antibodies can be formed with non-random orientation[22,23]. The biochemical modification on the surface of MEA may cause the fouling, and lead to a reduction of the electrochemical response current $[2,6]$, so the SAMs is formed on the inner surface of reaction channel in the cover plate.

\section{Immobilization of the Capture Antibody}

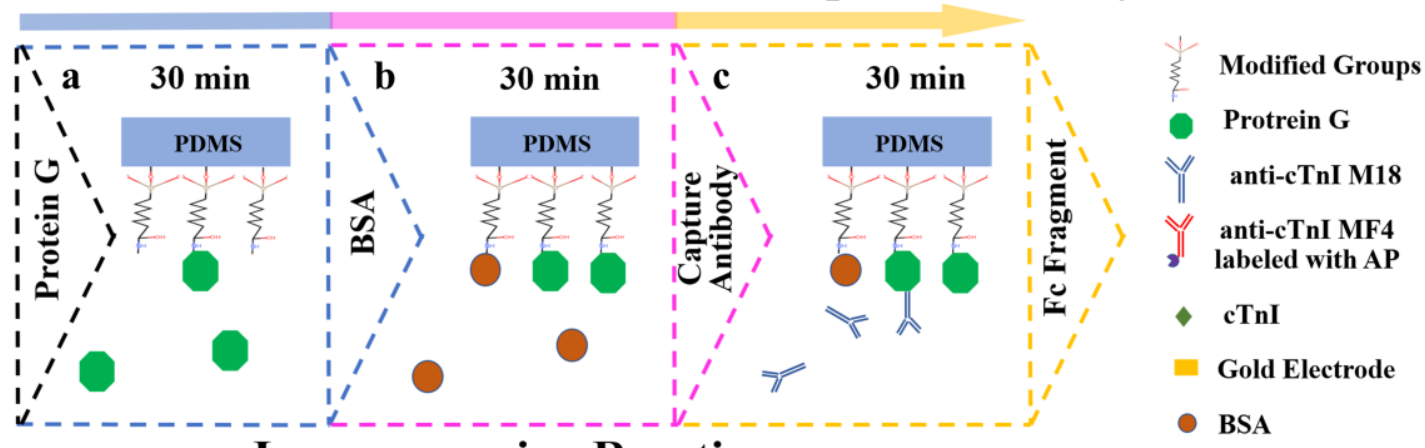

Immunosensing Reactions

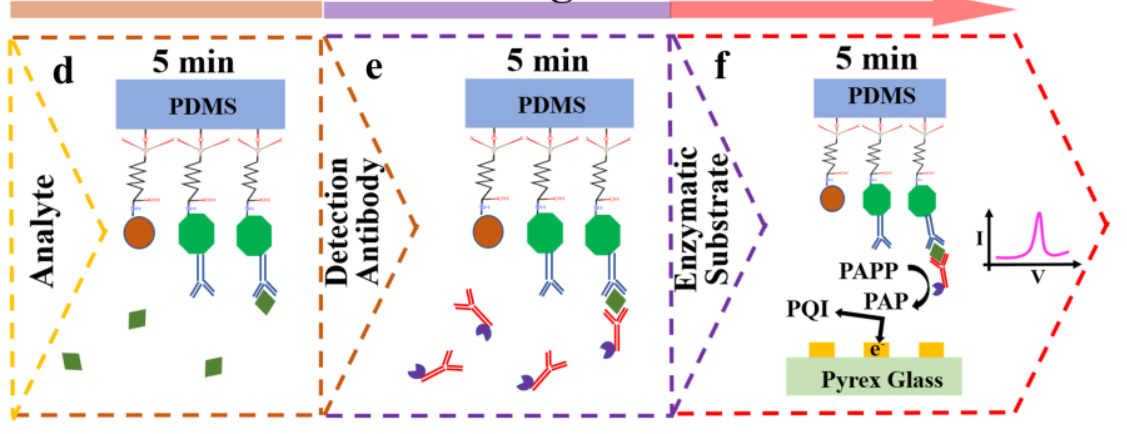

Fig. 1. The formation process of SAMs and the immunosensing reactions in $\mu$ FED. a Formation of protein G layer. b Blocking with BSA. $\mathbf{c}$ Immobilization of capture antibody. $\mathbf{d}$ Recognition of analyte in sample. $\mathbf{e}$ Bonding with AP labeled antibody. $\mathbf{f}$ AP catalytic reaction.

Firstly, the inner surface of reaction channel is modified with the functional groups, which is ended with carboxyl groups and can bond with protein $\mathrm{G}$. The formation process of the functional groups is achieved by referring to the methods previously reported [6,24]. The detailed modification process of the chemical groups on PDMS cover plate is described in Supplementary Material. In order to mitigate the risks posed to operator, anhydrous ethanol instead of toluene is used as the organic solvent.

Secondly, the protein layers, especially the antibody layer, are immobilized on the inner surface of the reaction channel. A protein $G$ monolayer is formed before the antibody is immobilized on the surface. Because the protein $\mathrm{G}$ can only bond with the $\mathrm{F}_{\mathrm{c}}$ portion of the IgG monoclonal antibody specifically, so that the M18 monoclonal anti-cTnI antibodies could be immobilized and their $\mathrm{F}_{\mathrm{ab}}$ portions could point to the solution with proper orientation. The protein $\mathrm{G}$ solution about $500 \mu \mathrm{g} / \mathrm{ml}$ in PBS is injected into the channel and incubated for 30 min such that free amino groups on the protein G's surface can react with the activated carboxyl groups (as shown in Fig.1a). After the reaction channel is rinsed with PBS for removing the unbound protein $\mathrm{G}$, the bovine serum albumin (BSA) solution, about $1 \mathrm{mg} / \mathrm{ml}$ in PBS, is injected and incubated for 30 min to block the unbound carboxyl groups (as shown in Fig.1b), and then rinsed with PBS again. Furthermore, the M18 anti-cTnI antibody solution about $200 \mu \mathrm{g} / \mathrm{ml}$ in PBS is injected into the reaction channel and incubated for about $30 \mathrm{~min}$, and then the channel is rinsed with PBS, as shown in Fig.1c. After that, the reaction channel is blow- dried by nitrogen, and stored at $4{ }^{\circ} \mathrm{C}$ 
before use.

The modified surface was characterized with atomic force microscope (AFM), the formed SAMs would cause the surface roughness change. As to further verify the immobilized SAMs, especially the capture antibody layer, the fluoroisothiocyanate-labeled immunoglobulin G (IgG-FITC) was adopted as an optical marker and the solution about $200 \mu \mathrm{g} / \mathrm{ml}$ was injected into the channel as a substitution for the capture antibody. To ensure the reliability, the IgG-FITC and the capture antibody should be both murine monoclonal IgG antibodies. This SAMs was similar to the structure shown in Fig.1c. After rinsed with PBS solution several times, the IgG-FITC modified channel was measured with a laser scanning confocal microscope (LSCM). The blue laser with the wavelength of $488 \mathrm{~nm}$ was used to excite the yellow-green fluorescence of IgG-FITC with the wavelength of 500 535 nm. The influence of the modification treatments on the electrochemical response signal of MEA was tested in a solution of $0.5 \mathrm{mM} \mathrm{K}_{4}\left[\mathrm{Fe}(\mathrm{CN})_{6}\right]$ and $0.1 \mathrm{M} \mathrm{KCl}$ by $\mathrm{CV}$ method at a scan rate of $100 \mathrm{mV} / \mathrm{s}$. The immobilization of the capture antibody on the inner surface of the PDMS channel and the immune recognition ability for the cTnI were further tested by the immunoassay and electrochemical measurements. The cTnI solution, which is $1 \mu \mathrm{g} / \mathrm{ml}$ in PBS, solution of detection antibody labeled with AP and the solution of enzyme substrate were injected and incubated in sequence within a modified $\mu \mathrm{FED}$. The catalyzed substrate was tested by $\mathrm{CV}$ and differential pulse voltammetry (DPV) respectively. The bare PDMS channel and gold MEA were also tested as the control.

Electrochemical immunoassay of $\mathbf{c T n I}$ in the $\boldsymbol{\mu F E D}$ with serum samples The immunoassays are applied in the $\mu$ FEDs with the Differential Pulse Voltammetry (DPV) technique, and the analyte sample is prepared by human serum and recombinant human troponin I antigen. The analyte sample is injected into the reaction channel and incubated for $5 \mathrm{~min}$, so that the $\mathrm{cTnI}$ in the analyte solution can be specifically recognized and captured by the immobilized M18 anti-cTnI antibody layer (as shown in Fig.1d). After the reaction channel is rinsed by PBS to remove the unbound antigen, the MF4 anti-cTnI antibody labeled with AP about $100 \mu \mathrm{g} / \mathrm{ml}$ in $10 \mathrm{mM}$ Tris- $\mathrm{HCl}$ at $\mathrm{pH} 8.5$ is injected into the reaction channel (as shown in Fig.1e), and the Tris- $\mathrm{HCl}$ solution is injected for rinsing after the antibody solution was incubated for $5 \mathrm{~min}$. Finally, the PAPP solution about $4 \mathrm{mM}$ in Tris- $\mathrm{HCl}$ is injected into the reaction channel. The PAPP could be converted to the electrochemical active species 4-aminophenol (PAP) by $\mathrm{AP}$, which is labeled on the antibody. The PAP can be oxidized into p-quinoneimine (PQI) on the surfaces of the electrodes at certain potential, and a current peak can be detected (as shown in Fig.1f). After the substrate solution is incubated for $5 \mathrm{~min}$, the $\mu \mathrm{FED}$ is connected to the electrochemical station through an USB female plug. During the DPV measurement, the potential is scanned from $-0.1 \mathrm{~V}$ to $+0.4 \mathrm{~V}$ vs. the screen-printed reference electrode (SPRE) in $3 \mathrm{M} \mathrm{KCl}$ solution, with $6 \mathrm{mV}$ pulse increasement, 50 $\mathrm{mV}$ pulse amplitude and $60 \mathrm{mS}$ pulse width. The supporting electrolyte chamber is fulfilled with the 3 $\mathrm{M} \mathrm{KCl}$ solution before the immunoassay, and the $2 \#$ inlet and outlet are sealed by electrostatic stickers for forming a flow dead zone. The cTnI immunoassays are applied in the concentration range of $10 \mathrm{pg} / \mathrm{ml}$ $\sim 1 \mu \mathrm{g} / \mathrm{ml}$, and a serum sample without $\mathrm{cTnI}$ is employed as the blank control. The immunoassay can be done in the $\mu \mathrm{FED}$ within about 15 minutes.

\section{Results and Discussions}

Simulation of the electrolyte chamber The concentration of the supporting electrolyte for the reference 
electrode is one of the key factors affecting the potential of the reference electrode, which directly affects the identification of the analyte. Typical commercialized reference electrode is always encapsulated in a glass/Teflon tube which is fulfilled with the supporting electrolyte solution, and the electrolyte solution in tube is always isolated from the sample solution by an ion-exchange porous glass/ceramic plug or a salt bridge, to avoid the pollution and dilution. It is not only complex and uneconomic to realize a glass/ceramic layer or porous structures in a microfluidic device, but also limits the application and integration of electrochemical sensor in a microfluidic device [25]. The laminar flow condition always occurs in microchannels, due to the low Reynolds number which could be inferred from the small width or height of the microchannel, so that the mass transfer between different solutions is only dominated by diffusion without the convection or turbulent flow. Based on this phenomenon, we speculated that the decrease of concentration in an independent electrolyte chamber might be inefficient and tolerable, which was aroused by the injected solution in reaction channel.

The concentration distribution in the electrolyte chamber during the electrochemical immunoassay process were simulated and analyzed with COMSOL. A 2D model was configurated and referred to the microfluidic network in PDMS cover plate. The electrolyte chamber was fulfilled with a concentrated solution in water about $3 \mathrm{M}$ to mimic the $\mathrm{KCl}$ supporting electrolyte. The pure water flew through the reaction channel with a velocity of $2 \mathrm{~mm} / \mathrm{s}$ to mimic the solution injected during immunoassay. The mass transfer process in the microfluidic network was studied with Laminar Flow (spf) and Transport of Diluted Species (chds) modules.

The concentration distribution figures at different times are illustrated in Fig.2a, and the concentration variations were profiled along the yellow dash line, which indicates the edge of SPRE near the intersection of the channel and chamber, the concentration curves are shown in Fig.2b. The maximum decrease of concentration in the chamber was about $0.6 \mathrm{M}$ in $15 \mathrm{~min}$. Based on the calculation of Nernst equation, the variation of the potential aroused by the change of the concentration should be smaller than $7 \mathrm{mV}$ and was tolerable.
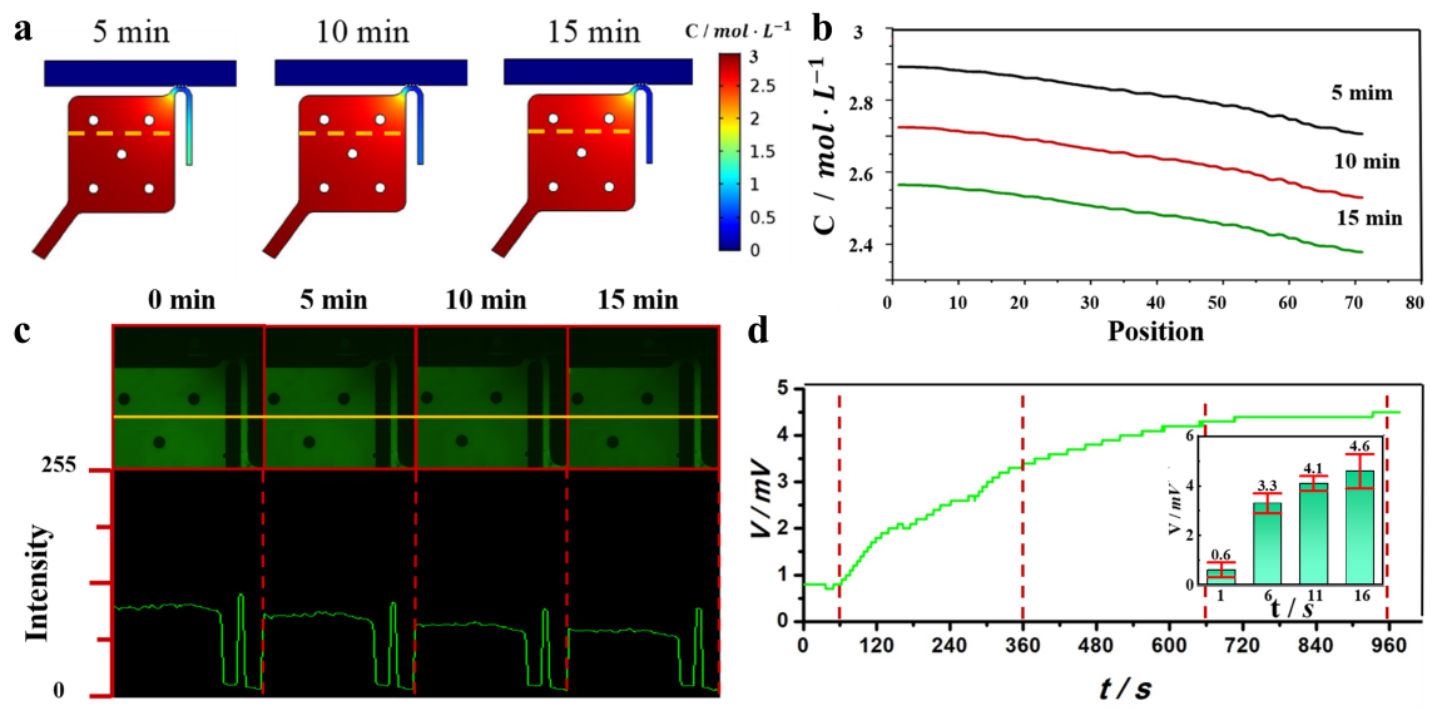

Fig. 2. The verification of the miniaturized reference electrode. a The simulation of mass transfer process in the electrolyte chamber. b The concentration profiles along the yellow dash line at various time points. $\mathbf{c}$ The fluorescence micrographs and the fluorescence intensity profiles of the electrolyte chamber at various time points. d The OCPT diagram of SPRE vs. CHI 111, inset: the histogram of potential difference at various time points, the error bars correspond to the standard deviation ( $\mathrm{n}=5$ ). 
Verification of SPRE in $\boldsymbol{\mu F E D}$ The performance of SPRE without the electrolyte chamber was verified by measuring the $\mathrm{OCP}$ (open circuit potential) in $3 \mathrm{M} \mathrm{KCl}$ solution with a commercial $\mathrm{Ag} / \mathrm{AgCl}$ reference electrode (CHI 111). The measurements were applied with 5 SPREs respectively and repeated twice. The potential difference was smaller than $3 \mathrm{mV}$ invariably. We also found that the color of SPREs varied from silver gray to dark brown, after the oxygen plasma treatment. The color of SPRE could not return to silver gray by rinsing with $\mathrm{HCl}$ solution, but by rinsing with alcohol. And the OCP between the color changed SPRE and CHI 111 was almost unchanged and still smaller than $3 \mathrm{mV}$. It could be inferred that the color change of SPRE might be caused by the ashed colloid in the $\mathrm{Ag} / \mathrm{AgCl}$ ink, rather than the oxidation of Ag.

The performance and the stability of the SPRE assembled in $\mu$ FED was tested by observing the fluorescence intensity distribution in the microfluidic network with an inverted fluorescence microscope and recording the potential variation of SPRE vs. CHI 111 with Open Circuit Potential-Time (OCPT) technique. In the fluorescent observation, the electrolyte chamber was fulfilled with the solution of $3 \mathrm{mM}$ fluorescein sodium in DI water. In the OCPT test, the chamber was fulfilled with $3 \mathrm{M} \mathrm{KCl}$ solution, and the SPRE connected with the CHI 111 by an injection tube through 2\# inlet. During these measurements, the DI water was continuously injected into the reaction channel to mimic the injection processes in immunoassay.

The fluorescence micrograph and the fluorescence intensity profile were recorded every 5 minutes at the same position as in the simulation, and the fluorescent images were processed with Image-Pro Plus, as shown in Fig.2c. In the first 5 min, the decrease in the fluorescence intensity was negligible. After 10 min, the fluorescence intensity near the intersection was lower slightly. The decrease in the fluorescence intensity after 15 min was visible, but still less than $30 \%$. It could be inferred that the dilution of the electrolyte in the chamber during the electrochemical immunoassay would be tolerable. The tolerable decrease of the electrolyte could also be confirmed by the OCPT diagram shown in Fig. $2 \mathrm{~d}$. In the first minute, the potential difference between the SPRE and CHI 111 was recorded without any interference, and the difference was always smaller than $1 \mathrm{mV}$. So that the potential accuracy of the SPRE could be validated. After one minute, the injection of DI water was started, and the potential difference was increasing. Because the potential of the SPRE would be increasing with the decreasing of the concentration of $\mathrm{KCl}$ solution in electrolyte chamber, which could be derived from the Nernst equation. The acceleration became slower with time. It could be explained by the decline of concentration difference between electrolyte chamber and reaction channel and the decrease of the falling rate of concentration in chamber. Even the potential difference was increasing, it was still smaller than $6 \mathrm{mV}$ in 15 minutes, and the potential variation of the SPRE was tolerable. Thus, the efficiency and stability of the SPRE were verified, and it could afford the demand of $\mu$ FED.

The optimization of the interstice in MEA The curves about the CV tests of MEA at different scan rate were shown in Fig.S3a, and the peak current density increased with the accelerated scan rate. A linear relationship could be found between oxidation peak and the square root of scan rate in the range of 10$200 \mathrm{mv} / \mathrm{s}$ (Fig.S3b). Thus, the electrochemical reaction process on the surface of MEA is controlled by the diffusion of the reactant.

In order to find the optimal $W_{i}$, a group of $\mu$ FEDs were tested in a solution of $0.5 \mathrm{mM} \mathrm{K} 4\left[\mathrm{Fe}(\mathrm{CN})_{6}\right]$ and $0.1 \mathrm{M} \mathrm{KCl}$ by cyclic voltammetry (CV) method at a scan rate of $100 \mathrm{mV} / \mathrm{s}$. Each MEA is consisted of 100 strip electrodes, and the width of each electrode $\left(W_{e}\right)$ is $10 \mu \mathrm{m}, W_{i}$ varies and the superficial area 
of MEA is $0.5 \mathrm{~mm}^{2}$ (the side wall surface area of MEA is ignored). The measurements were repeated for 5 times, and the cyclic voltammograms are shown in Fig.3a. The oxidation peak current density $\left(J_{p}\right)$ increased significantly with the enlargement of $W_{i}$, and the acceleration of $J_{p}$ got slower and slower. It could be explained by the cylindrical distribution of diffusion field at the edges of MEA. The variation of the overlapping region, belongs to adjacent electrodes, got smaller with the increasement of $W_{i}$, and the shielding effect was eliminated gradually. Furthermore, the increasement of $J_{p}$ tended to be ignorable when $W_{i}$ is longer than $40 \mu \mathrm{m}$, and the cumulative increasement is less than $5 \%$ of the maximum $J_{p}$. It could be inferred that the overlaps of the diffusion fields might have vanished beyond this distance. Thus, we surmised that the optimal $W_{i}$ should be between $30 \mu \mathrm{m}$ and $40 \mu \mathrm{m}$, and it should be the distance where the adjacent diffusion fields were tangent. And the optimal $W_{i}$ for this group of MEAs could be estimated as $34 \mu \mathrm{m}$ by finding the inflection point of linear fitting curves of the $J_{p}$ plots in different sections. It could also be found that $J_{p}$ of MEA without shielding is obviously bigger than that of macroscopic plate electrode which is $1.09 \mu \mathrm{A} / \mathrm{mm}^{2}$ based on the calculation of Randles-Sevick equation. The discrepancy may be aroused by the largely increased length of edge in MEA.

In order to verify whether the optimal $W_{i}$ is affected by $W_{e}$, another group of the MEAs, of which $W_{e}$ are $20 \mu \mathrm{m}$, were also tested with the same method. To keep the total superficial area of MEA constant, each MEA consists of 50 strip electrodes. The cyclic voltammograms are shown in Fig.3c, when $W_{i}$ is larger than $40 \mu \mathrm{m}$, the change of $J_{p}$ tends to be ignorable. It could be also found that the maximum $J_{p}$ in the group of $10 \mu \mathrm{m}$ in $W_{e}$ is bigger than in the group of $20 \mu \mathrm{m}$, although the total surface area of MEA keeps still. That might be caused by the increased quantity of edge in the MEA with thinner $W_{e}$, and the more quarter-cylindrical efficient diffusion fields contributed to the elevation of $J_{p}$. The optimal $W_{i}$ for this group of MEAs could be also estimated by calculating the intersection of linear fitting curves, and the result was $35 \mu \mathrm{m}$. The difference between the results of $W_{i}$ in these two groups is not obvious $(1 \mu \mathrm{m})$. Thus, we supposed that the optimal $W_{i}$ for MEAs is inapparently affected by $W_{e}$, and the distribution of the diffusion zone around the edge in different optimal MEAs shall be similar. In other words, the current generated by the edge effect, which is a component of total current of MEA, shall be proportional to the length of the edge, and its density $\left(J_{e}\right)$ shall be relatively stable. Thus, $J_{p}$ of MEA can be described as the equation:

$$
J_{p}=\left(J_{S} \cdot A+J_{e} \cdot L\right) / A=\left[J_{S} \cdot\left(W_{e}+2 T\right) \cdot \mathrm{L}+2 J_{e} \cdot L\right] / A=J_{S}+2 J_{e} /\left(W_{e}+2 T\right)
$$

where $J_{s}$ is the density of the current generated by the plane surface of the MEA, $A$ is the total surface area of MEA, and $L$ is the summation length of all edges in MEA, and $T$ is the thickness of conductive metal layers of MEA. Solving the equation by substituting the data without shielding effect, $J_{s}$ and $J_{e}$ shall be $1.15 \mu \mathrm{A} / \mathrm{mm}^{2}$ and $11.78 \mathrm{nA} / \mathrm{mm}$ respectively. Because this electrochemical reaction process is controlled by the diffusion of the reactant, $J_{s}$ and $J_{e}$ shall be functions of the scan rate. $J_{s}$ is close to the result of Randles-Sevick equation $\left(1.10 \mu \mathrm{A} / \mathrm{mm}^{2}\right)$, but slightly bigger. The deviation may be caused by the interaction between the plane diffusion field and the cylindrical diffusion region, which generated by the surface and edges of the electrode respectively. A gradual transition region is formed at their junction, and its efficiency is between both. This equation can be used for the estimation of the current density of the MEA with different $W_{e}$. 

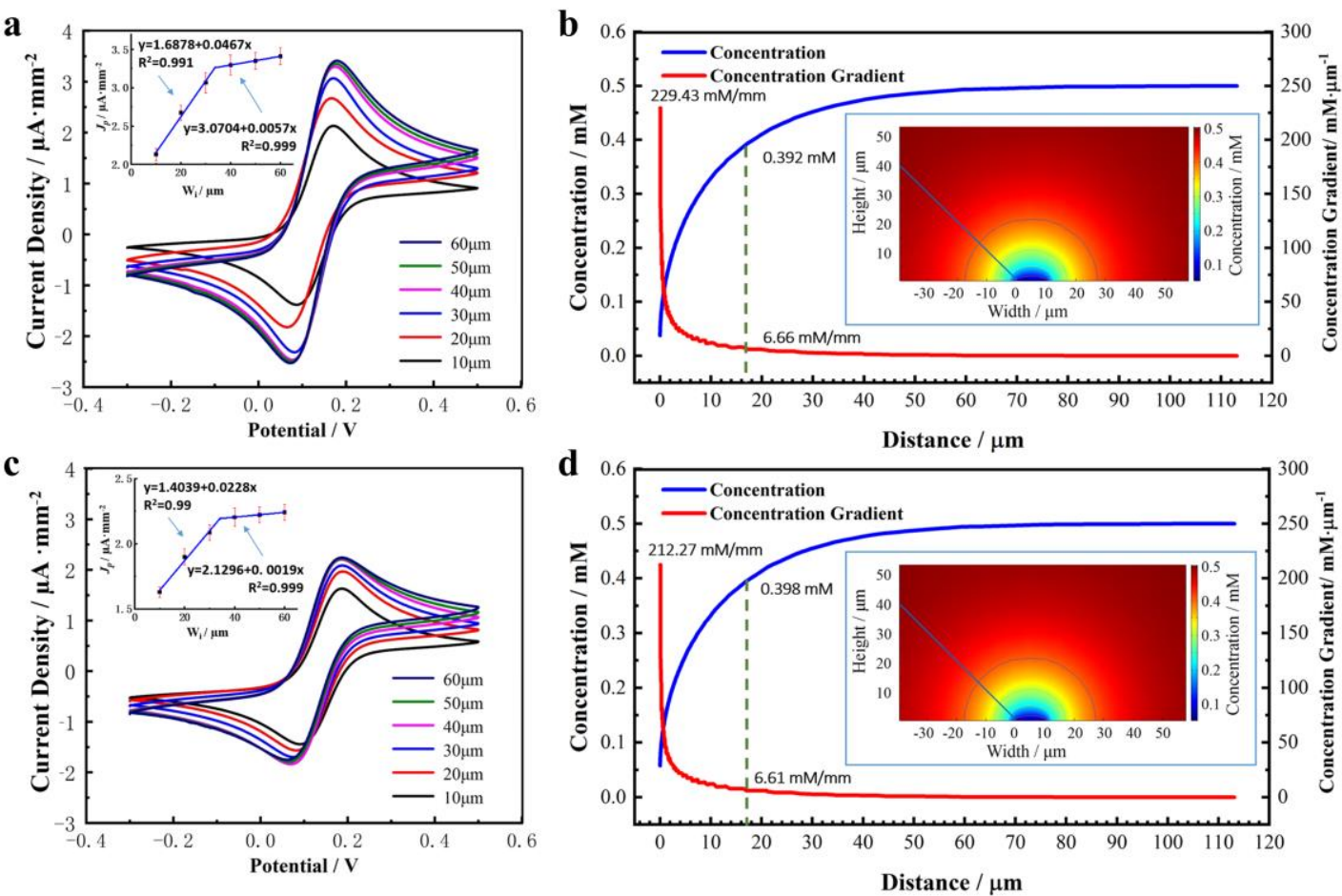

Fig. 3. The CV tests of MEA with different parameters and simulation of concentration field. a c The Cyclic voltammograms of the MEAs recorded in a solution of $0.5 \mathrm{mM} \mathrm{K}_{4}\left[\mathrm{Fe}(\mathrm{CN})_{6}\right]$ and $0.1 \mathrm{M} \mathrm{KCl}$ at the scan rate of $100 \mathrm{mV} / \mathrm{s}$, and the insets describe the variation of the peak current vs. $W_{i}$. b d The simulation diagrams about the diffusion field distribution of single electrode at the moment the peak current appearing, and the curves describe the variation of concentration and concentration gradient along the blue line in the inset. The $W_{e}$ of the MEAs tested in $\mathbf{a}$ and $\mathbf{b}$ is $10 \mu \mathrm{m}$, and in $\mathbf{c}$ and $\mathbf{d}$ is $20 \mu \mathrm{m}$, the error bars correspond to the standard deviation $(\mathrm{n}=5)$.

We also attempted to further explain the optimal $W_{i}$ from the view of diffusion field of the electrochemical substance. We anticipated that the optimal Wi should be exactly equal to twice of the diffusion field thickness where the shielding effect was totally eliminated. The concentration distribution and concentration gradient were analyzed with the 2D simulation model. The theorical maximum peak current density of the MEA could be calculated by the simulated peak current of single electrode without any shielding. The simulated peak current density of $10 \mu \mathrm{m}$ wide electrode is about $3.56 \mu \mathrm{A} / \mathrm{mm}^{2}$, which was very close to the data we measured in experiments (the average value is about $3.43 \mu \mathrm{A} / \mathrm{mm}^{2}$ ), thus, the simulation model was valid. The simulation results about concentration distribution and concentration gradient near the surface of the electrode, which is $10 \mu \mathrm{m}$ wide, are shown in Fig. $3 \mathrm{~b}$. The curves, which were drawn in the inset, described the concentration distribution and concentration gradient at the position labeled by the blue line, based on the simulation data at the peak current time point. It could be found that the concentration and its gradient were changing drastically near the surface of the electrode. At the position $17 \mu \mathrm{m}$ away from the surface of the electrode, which is half of the optimal $W_{i}$ we deduced, the concentration gradient was $6.66 \mathrm{mM} / \mathrm{mm}$ (about $3 \%$ of the gradient at the surface), and the concentration was about $0.392 \mathrm{mM}$ (about $80 \%$ of the original solution). The diffusion of the electrochemical active substance was still existing, so that the boundary of the diffusion field should not be at this position. Beyond this distance, the increasing rate of concentration and the decreasing rate of concentration gradient were approaching to zero gradually slower, until the distance of $105 \mu \mathrm{m}$, where the actual boundary of the diffusion field should be. It indicated that the mass transfer efficiency in this 
section was obviously lower than the inner section. Thus, the increasement of the current tended to be ignorable, when the interstice was longer than the optimal $W_{i}$ we deduced. We also tested a MEA without shielding effect, of which $W_{i}$ is $210 \mu \mathrm{m}$, to verify the maximum response current without shielding effect, and the current was about $3.45 \mu \mathrm{A}$, which was only about $0.05 \mu \mathrm{A}$ higher than the maximum value we measured above. For the MEA whose $W_{i}$ is $34 \mu \mathrm{m}$, its response peak current should be $3.27 \mu \mathrm{A}$, based on the calculation of the linear equation shown in the inset of Fig.3a. It is about $5 \%$ smaller than the current tested without shielding effect. Meanwhile, the fluidic example consumption needs to be quadruple to eliminate the inconspicuous difference. Thus, it is unnecessary to vanish the shielding effect entirely, and the partly overlap in inefficient diffusion fields is tolerable.

We tried to find a criterion for the estimation of optimal $W_{i}$, which should be twice of the radius of the efficient diffusion zone. The diffusion field of the electrode which is $20 \mu \mathrm{m}$ in $W_{e}$ was also analyzed, as shown in Fig.3d. The concentration and the concentration gradient at the distance about $17.5 \mu \mathrm{m}$ away from the surface were analyzed, and the distance is the half of the deduced optimal $W_{i}$. The concentration was $3.98 \mathrm{mM}$ and close to $80 \%$ of the original concentration. The concentration gradient was 6.61 $\mathrm{mM} / \mathrm{mm}$ and was approximately equal to $3 \%$ of the gradient at the surface $(212.27 \mathrm{mM} / \mathrm{mm})$. The value of these parameters was similar to the group of MEAs whose $W_{e}$ was $10 \mu \mathrm{m}$. Thus, we assume that the concentration (about $4 \mathrm{mM}$ or $80 \%$ of the original) or the concentration gradient (about $6.6 \mathrm{mM} / \mathrm{mm}$ or $3 \%$ of the gradient at the surface) could be considered as the criterion for the optimal $W_{i}$.

In order to verify whether the absolute or the relative value of the criteria is more effective, we also analyzed the diffusion field with the initial concentration of $5 \mathrm{mM}$, and the result is shown in Fig.S4. Although the concentration and gradient are 10 times bigger at each point, the diffusion field diagram and the curves of concentration and concentration gradient are similar to those in $0.5 \mathrm{mM}$. Thus, the criterion in the relative value form is more appropriate.

The precision of the candidate criterions at different scan rate was further verified, and the peak oxidation current density of the MEAs with different $W_{i}$ were drawn in Fig.4a. The difference value between the current density of the MEAs, whose $W_{i}$ is $60 \mu \mathrm{m}$ and $210 \mu \mathrm{m}$, is ignorable. Thus, in the measurements of the MEA with $210 \mu \mathrm{m}$ in $W_{i}$, the shielding effect might not exist, and the current density could be considered as the maximum limit at certain scan rate. Meanwhile, with the acceleration of the scan rate, the current density of MEA could approach the maximum limit with smaller $W_{i}$. As a roughly estimate, $95 \%$ of maximum current density was used to distinguish the gently and sharply increasing zones. The data in different zones were linear fitted respectively, and the $\mathrm{R}^{2}$ of these fitting curves were all bigger than 0.99 . The slope of the fitting lines in the sharply increasing zones were significantly bigger than in the gently increasing zones. The optimal $W_{i}$ was deduced by finding the intersection of the fitting lines. The curves of the measurements at a scan of $1000 \mathrm{mV} / \mathrm{s}$ are an exception. When $W_{i}$ was bigger than $20 \mu \mathrm{m}$, the difference of current density did barely increase with the increase of the $W_{i}$ (the slope of the line is 0.00016 ). The optimal $W_{i}$ at this scan rate was estimated by plugging the $95 \%$ of the maximum current $(6.20 \mu \mathrm{A})$ into the fitting equation of the smallest two points. The distribution of diffusion field of MEA at different scan rate was analyzed with the established simulation model, and the optimal $W_{i}$ was also estimated with the candidates of criterion. The fitting curves of the estimated optimal $W_{i}$ were shown in Fig.4b, the estimated optimal $W_{i}$ has an inverse proportional relation with the root of the scan rate. The estimated points were close to the experimental data with perceptible error, and the trends of the fitting curves were consistent with the experimental result. The error between the experimental data and the estimation curve based on the concentration gradient is smaller than the estimation curve based on concentration. In addition to the measurement error in the experiment, the perceptible error may be 
led by the neglect of the shielding effect caused by the overlap of inefficient diffusion field. Even it is ignorable, the superposition of multiple resulted in a perceptible error in MEA. By comparing the residual sum of squares (RSS) between the optimal $W_{i}$ estimated by the criterion and deduced based on the experiments, it could be found that the estimation determined with concentration gradient $(\mathrm{RSS}=23.11)$ was more accurate than determined with the concentration $(\mathrm{RSS}=168.51)$.
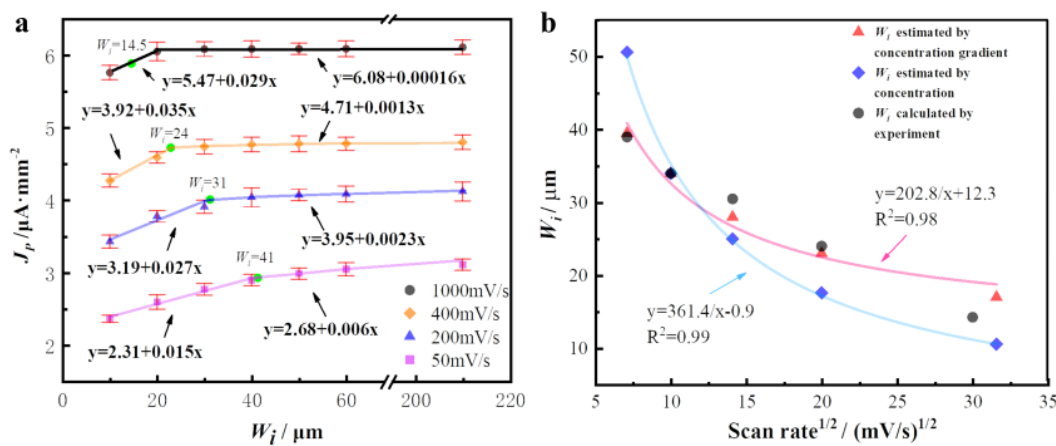

Fig. 4. The verification of the criterion for the optimal $W_{i}$. a The analyzing of the optimal $W_{i}$ at different scan rate $\left(W_{e}=10 \mu \mathrm{m}\right.$, $\mathrm{n}=5$ ), and the green points are the intersection points of fitting lines, the error bars correspond to the standard deviation $(\mathrm{n}=5)$. $\mathbf{b}$ The error analysis of the optimal $W_{i}$ estimated with different criterions

Based on the above analysis, the optimal $W_{i}$ should be twice of the radius of efficient diffusion zone, and the boundary of the efficient diffusion zone could be estimated by finding the position where the concentration gradient decrease to $3 \%$ of the maximum gradient at the surface of electrode. Meanwhile, the optimization method eliminated the shielding effect by changing the interstice of the MEA, and it would not conflict with other modification methods on the electrode (e.g. metal nanoparticles[19], graphene[2] and covalent organic frameworks[26]), which are approaches to elevate the performance of the electrode by increasing of the electron transfer rate. It is expected to further improve the performance of MEA by applying the optimization and modification methods simultaneously.

Verification and optimization of the modified SAMs To characterize the SAMs, a modified PDMS cover plate (shown in Fig.1c) was observed with an AFM in a scan area of $2 \mu \mathrm{m} \times 2 \mu \mathrm{m}$, and an original PDMS cover plate was also observed as a control. The AFM images of the original and the modified plates were illustrated in Fig.5a and b, the modified surface was obviously more irregular than the original. The average surface roughness $\left(\mathrm{R}_{\mathrm{a}}\right)$ was also measured by the AFM. The $\mathrm{R}_{\mathrm{a}}$ of the modified surface was about $2.58 \mathrm{~nm}$ and much bigger than the original surface, which was about $0.779 \mathrm{~nm}$. It could be inferred that the SAMs of M18 antibody, Protein G and silane were formed on the inner surface of the reaction channel.

The fluorescent images of the original channel and the modified channel were shown in Fig. $5 \mathrm{c}$ and d, the borders of the channels were indicated by red dash lines, and the yellow lines indicated the positions where the fluorescent intensity were recorded. Barely fluorescent could be observed in the original channel, and the negligible distinction of intensity between inside and outside the channel might be caused by the few non-specific absorptions of IgG-FITC on the inner surface of the channel. In the measurement of modified channel with the same parameters, an appreciable fluorescent image could be observed, the intensity in the channel was obviously stronger than in the original channel. The stable immobilization of the IgG-FITC on the inner surface of the PDMS channel could be confirmed. Because the IgG-FITC and capture antibody are both murine monoclonal IgG antibodies, and they all can bond 
with protein $G$ through their similar $F_{c}$ portion. It could be inferred that the capture antibody could be also immobilized on the inner surface of the reaction channel with this method.

Though we tried to form the SAMs on PDMS to prevent fouling of MEA, the EDC and Sulfo-NHS solution applied for activating the carboxyl groups, which was also commonly used in the biochemical modification on gold [27,28], may cause the unexpected protein bonding on the surface of gold MEA inevitably. The fouling of MEA by the unexpected bonded protein or groups may occlude the electron transfer between the electrodes and solution and cause a decrease in current. The conjecture was supported by the $\mathrm{CV}$ tests in $\mathrm{K}_{4}\left[\mathrm{Fe}(\mathrm{CN})_{6}\right]$ solution shown in Fig.5g, the response current of the MEA in the $\mu$ FED, which was modified after the cover and substrate plates were assembled, was apparently smaller than the MEA in the $\mu$ FED without any treatments. We tried to apply the modification method before the elements of the $\mu$ FED were assembled, but there was another new problem, as shown in eFig.5e. Although a measurable fluorescence could be observed in the channel, the fluorescence intensity was weakened, and the distribution became fragmented. The protein $\mathrm{G}$ layer might be partly damaged or deactivated by the oxygen plasma treatment which is commonly applied in the bonding process of PDMS and glass. We further attempted to coordinate the modification method and the bonding process. We also tried to reorganize the process. The functional group layers would be modified and activated before the bonding process, and the SAMs of protein was formed after the assembling of $\mu$ FED. The fluorescence images of these $\mu$ FEDs were similar to Fig. $5 \mathrm{~d}$, the damages of the protein by the oxygen plasma treatment was avoided by reorganizing the process sequence of modification method and bonding process. Meanwhile, their response current curves (shown in Fig.5g) were close to the bare gold MEA, which indicated the elimination of the surface fouling of MEA. These results demonstrated the better performance of the reorganized modification process. Blocking process during the formation of SAMs is vital for the eliminating of nonspecific recognition of the analyte antigen or the labeled detection antibody. The potential unbonded sites on the functional group layer should be blocked, and the blocking agent should not bond with protein $G$ or obstruct the bond between protein $G$ and antibody. The BSA solution we adopted is a widely used blocking agent not only in SAMs, but also in ELISA or western blot tests[29,30]. In a modified $\mu$ FED which was blocking for 4 hours, its fluorescent image (shown in Fig.5f) was significantly darker than the $\mu$ FED which was blocking for 30 minutes (Fig.5c). We also repeated the experiment with the BSA which is $\mathrm{IgG}$ free. Although the blocking time is longer, its fluorescent image (shown in Fig.5i) was a little brighter than the $\mu$ FED which was blocking with the BSA in reagent grade (Fig.5d), and the distribution was more even with less black patches. That might be caused by the small amount of IgG within the BSA in reagent grade, the inartificial IgG in BSA would occupy appreciable number of binding sites on protein $G$ monolayer, before the capture antibody monolayer was formed. The application of BSA IgG free in blocking process could reduce the occupied sites of inartificial IgG on the protein $\mathrm{G}$ layer and improve the immobilization of anti-cTnI capture antibody. 

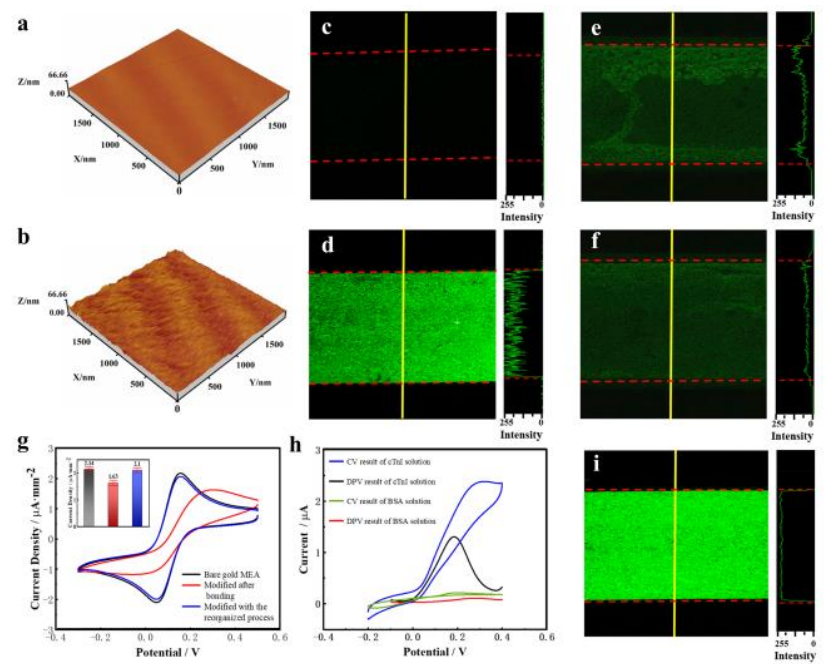

Fig. 5. The characterization of the modified surface. a c The AFM microscopic image and LSCM image of the original PDMS cover plates, the red dash line labeled the edges of the channel and the yellow line shown the position where the fluorescence intensity was measured. b d The AFM microscopic image and LSCM image of the modified PDMS cover plates. e The SAMs damaged by oxygen plasma. $\mathbf{f}$ The modified surface which is blocked for 4 hours. $\mathbf{g}$ The voltammetry curves of bare gold MEA, the MEA polluted by the modification process, and the MEA in the $\mu$ FEDs modified with the reorganized process, inset described the statistic value of the anode peak current, the error bars correspond to the standard deviation ( $\mathrm{n}=5)$. $\mathbf{h}$ The CV and DPV tests with analyte sample, and the BSA solution is employed as control. $\mathbf{i}$ The LSCM image of the PDMS surface modified with the reorganized process.

The immobilization and recognition ability of cTnI of the capture antibody was further demonstrated by the immunoassay and electrochemical measurements. The response current of the $\mu$ FED, which was injected with cTnI solution, was significantly increased, and its curve was obviously different from the $\mu$ FED which was injected with BSA solution, no matter in the CV or DPV tests (shown in Fig.5h). This result illustrated the substrate could be converted into electrochemical reactant with the injection of cTnI solution during the immunosensing. cTnI in sample solution was recognized specifically by the immobilized antibody monolayer on the inner surface of the reaction channel. In the control group, the sandwich structure (capture antibody-analyte-detection antibody) could not constructed on the inner surface of reaction channel without cTnI, thus the substrate could not be converted without the enzyme labeled on detection antibody. Additionally, the background current measured by DPV method is smaller than $\mathrm{CV}$ method, and the current peak is also more obvious in voltammetry curve. Thus, the $\mu$ FEDs would be tested by DPV method in following electrochemical immunoassay

Validation of the immunoassay performance In the $\mu$ FED which was adopted for the immunoassay of cTnI, the MEA consists of 100 strip electrodes, and the width of each electrode $\left(W_{e}\right)$ is $10 \mu \mathrm{m}$. The interstice between electrodes $\left(W_{i}\right)$ is selected with the optimization method. The curve of the potential applied in DPV could be approximately considered as a single segment in CV, the optimization method should be valid in DPV with the similar scan rate. The PAP is the reactant directly involved in the electrochemical reaction, and its diffusion coefficient $\left(1.18 \times 10^{-5} \mathrm{~cm}^{2} / \mathrm{s}\right)$ is estimated by the Wilke-Chang equation. If all the cTnI molecules in the sample could be captured, the maximum concentration of PAP should be approximately equal to $0.28 \mathrm{mM}$, which is calculated based on the enzyme activity. The diffusion field is also analyzed with the simulation model, as shown in Fig.S5. The boundary of the 
efficient diffusion field could be estimated by the criterion we demonstrated. At the position about 17.9 $\mu \mathrm{m}$ away from the electrode surface, the concentration gradient is about $3.48 \mathrm{mM} / \mathrm{mm}$, which is about $3 \%$ of that at the surface $(116 \mathrm{mM} / \mathrm{mm})$. And the $W_{i}$ in the MEA which is integrated in the $\mu \mathrm{FED}$ for the electrochemical immunoassay shall be no less than $35.8 \mu \mathrm{m}$ to reduce the shielding effect, which is double radius of the efficient diffusion field. Thus, the $W_{i}$ of the MEA is determined as $40 \mu \mathrm{m}$, with a margin of $10 \%$. The DPV voltammograms for human serum samples with different concentration of cTnI were shown in Fig.6a. The significant current peaks can be observed around the potential of $0.185 \mathrm{~V}$, which associated with the oxidation from PAP to PQI, and the peaks are proportional to the concentration of $\mathrm{cTnI}$ in serum samples. The voltammogram of the blank control sample is flat and significantly different from the samples with cTnI. It indicates that the cTnI in human serum samples can be recognized specifically and efficiently by the SAMs we modified on the inner surface of reaction channel, and the nonspecific binding was negligible. The calibration curve is illustrated in Fig.6b, each point is drawn based on the average of 5 tests with the same concentration of cTnI. These results could be linear fitted with a coefficient of determination $\left(\mathrm{R}^{2}\right)$ about 0.9911 , which demonstrated that the response current peaks are directly proportional to the logarithm of concentration of $\mathrm{cTnI}$ in the range between $50 \mathrm{pg} / \mathrm{ml}$ and $1 \mu \mathrm{g} / \mathrm{ml}$. The theoretical limit of detection (LOD) could reach $5 \mathrm{pg} / \mathrm{ml}$. In this study, LOD is calculated as the concentration that gives the response signal 3 times of the blank signal $(\mathrm{S} / \mathrm{N}=3)[31,32]$. The average of blank signal $\left(\mathrm{S}_{\mathrm{b}}\right)$ is $142 \mathrm{nA}$ and its standard deviation $\left(\mathrm{SD}_{\mathrm{b}}\right)$ is $18 \mathrm{nA}$, which are obtained by measuring 10 independent blank serum samples. LOD is calculated with the following equation, LOD $=\left(\mathrm{S}_{\mathrm{b}}+3 \mathrm{SD}_{\mathrm{b}}-\mathrm{a}\right) / \mathrm{b}$, where $\mathrm{a}$ is the intercept, $\mathrm{b}$ is the slope of the fitted line. The detection linear range and precision of $\mathrm{cTnI}$ with the $\mu \mathrm{FED}$ could meet the requirements of the diagnosis of AMI.

The specificity of the $\mu \mathrm{FED}$ is verified with the interference serum samples, which were prepared with cardiac troponin $\mathrm{T}(\mathrm{cTnT})$, creatine kinase (CK), and BSA, as shown in Fig.6c. The concentration of these samples are all $1 \mu \mathrm{g} / \mathrm{ml}$, and the serum is employed as the control. Each interference was tested with $5 \mu \mathrm{FED}$. The response signal in each interference sample is similar to the blank control, and is obviously smaller than the $\mathrm{cTnI}$ sample. The reproducibility is tested with $\mu$ FEDs which have been stored for some days, as shown in Fig.6d. In the tests with cTnI sample, the response signal of the $\mu$ FED would be relatively stable in two weeks, and would decrease significantly after 3 months. We also tested these $\mu \mathrm{FED}$ with $\mathrm{K}_{4}\left[\mathrm{Fe}(\mathrm{CN})_{6}\right]$ solution, the response signal decreased slightly (smaller than $2 \%$ ) and is relatively stable in 6 months. The performance of MEA is stable and reproducible. The significant decrease of response signal should be aroused by the inactivation of antibodies or enzymes. The reproducibility of the $\mu$ FED should be improved by finding the method for keeping the activity of antibodies or enzymes.

Because the immunoassay performance is not only controlled by the performance of the working electrode but also affected by the immunobinding ability of different monoclonal antibodies and different enzymatic activity of AP from different suppliers, etc. The performance of different electrodes are evaluated with the result tested in potassium ferricyanide or ferrocyanide solution, as shown in Table.1, all the efforts could lead to significant amplification in the response signal. The response signal of the optimized MEA is 3.12 times the macroscale electrode with the same surface area. Compared to the MEA described in [6], there is still a $22 \%$ increasement. The amplification caused by optimization is considerable, meanwhile, it would not lead to any increasement of electrode manufacturing cost and processes compared to the methods modified nano functional particles/complex on the electrode surface. 
a

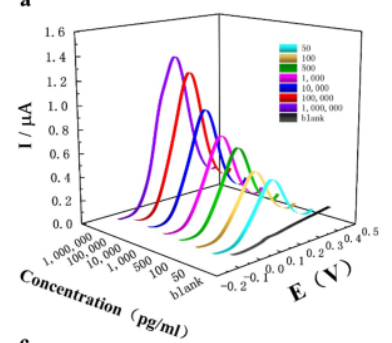

c

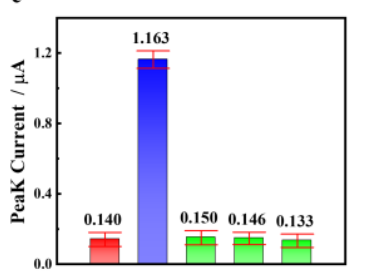

blank $\mathrm{CTnL}_{\mathrm{CTnT}} \mathrm{CK}$ BSA b

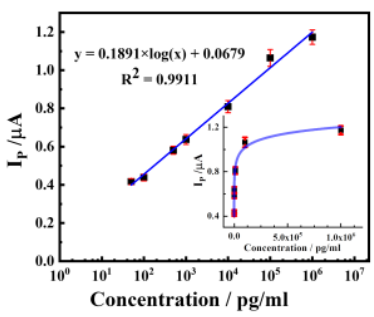

d

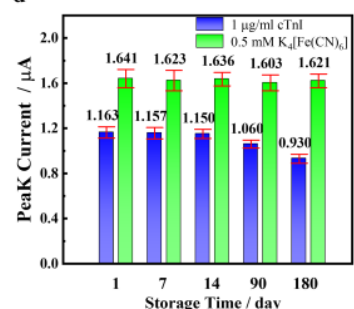

Fig. 6. The detection of the cTnI in serum sample. a The DPV voltammograms of analyte samples with cTnI in different concentration. b Calibration curves of cTnI between $10 \mathrm{pg} / \mathrm{ml}$ and $1 \mu \mathrm{g} / \mathrm{ml}$, the error bars correspond to the standard deviation $(\mathrm{n}=5)$. c The specificity tests, the concentration of each sample is $1 \mu \mathrm{g} / \mathrm{ml}$, and the human serum is employed as blank control $(\mathrm{n}=5)$. $\mathrm{d}$ The reproducibility is verified with cTnI sample $(1 \mu \mathrm{g} / \mathrm{ml})$ and $0.5 \mathrm{mM} \mathrm{K} \mathrm{K}_{4}\left[\mathrm{Fe}(\mathrm{CN})_{6}\right]$ solution $(\mathrm{n}=5)$.

Table 1 Comparison of the immunoassay performance of different electrochemical platforms for detection of cTnI

\begin{tabular}{ccccc}
\hline Electrode & Amplification factor ${ }^{*}$ & $\begin{array}{c}\text { LOD } \\
(\mathrm{ng} / \mathrm{ml})\end{array}$ & $\begin{array}{c}\text { Linear range } \\
(\mathrm{ng} / \mathrm{ml})\end{array}$ & Ref. \\
\hline MEA & - & 0.148 & $0.2 \sim 10^{4}$ & {$[6]$} \\
AuNPs/GCE & 1.1 & $10^{-6}$ & $5 \times 10^{-6} \sim 50$ & [33] \\
Optimized MEA & $1.22^{* *} ; 3.12^{* * *}$ & $5 \times 10^{-3}$ & $0.05 \sim 1000$ & This work \\
G-CNTs/GCE & 1.89 & $10^{-3}$ & $10^{-3} \sim 10$ & [34] \\
BNQDs/GCE & 4 & $0.5 \times 10^{-3}$ & $0.01 \sim 5$ & [35] \\
\hline
\end{tabular}

\footnotetext{
* The response current amplification factor between modified/optimized electrode and bare electrode tested in potassium ferricyanide/ferrocyanide solution; ${ }^{* *}$ The comparison between the optimized MEA and the MEA described in [5]; ${ }^{* * *}$ The comparison of between optimized MEA and macroscale band electrode; AuNPs/GCE: the glassy carbon electrode modified with gold nano particles; G-CNTs/GCE: the glassy carbon electrode modified with graphene and carbon nanotube hybrid; BNQDs/GCE: the glassy carbon electrode modified with boron nitride quantum dots.
}

\section{Conclusion}

We have presented a sensitive and portable $\mu \mathrm{FED}$ for the diagnosis of AMI, and the risk was evaluated by electrochemically measuring the concentration of cTnI. The interstice of MEA in the $\mu$ FED is optimized for eliminating the shielding effect, and the response signal of $\mu \mathrm{FED}$ is elevated significantly with a limited increasement in the volume of samples and reactants. We realized a stable and miniaturized reference electrode in the $\mu \mathrm{FED}$, and its tolerable reference potential deviation prevents the drift of response signal. And the immobilization process of the capture antibody is also reorganized to be compatible with bonding process. These efforts contributed to the improvement of the immunoassay performance of the $\mu \mathrm{FED}$ without any increasement of manufacturing cost and processes, and do not 
conflict with the modification of nano functional particles. The detection of cTnI could be completed in $15 \mathrm{~min}$, the response signal is linear to the logarithmic concentration of cTnI over $50 \mathrm{pg} / \mathrm{ml}$ to $1 \mu \mathrm{g} / \mathrm{ml}$, and the theoretical limit of detection is $5 \mathrm{pg} / \mathrm{ml}(\mathrm{S} / \mathrm{N}=3)$. The detection performance of the $\mu \mathrm{FED}$ is qualified for the immunoassay of $\mathrm{cTnI}$, and the operation process is simple enough for the people without medical professional skills. The development and integration of more microfluidic manipulation modules would further simplify the operation process. The $\mu$ FED is expected to be the substitute for ECG for the fast and accurate diagnosis of AMI, and it is possible to be applied even at home.

\section{Funding information}

This work is supported by National Natural Science Foundation of China (51875084), Science and Technology Planning Project of Dalian (1712032).

\section{Compliance with ethical standards}

The authors declare that they have no conflict of interest. All procedures performed in this work were in accordance with the protocols approved by the Biology and Medical Ethics Committee of Dalian University of Technology.

\section{References:}

1. Reiter M, Twerenbold R, Reichlin T, Haaf P, Peter F, Meissner J, et al. Early diagnosis of acute myocardial infarction in the elderly using more sensitive cardiac troponin assays. Eur Heart J.2011;32(11):1379-89.

2. Singal S, Srivastava AK, Gahtori B, Rajesh. Immunoassay for troponin I using a glassy carbon electrode modified with a hybrid film consisting of graphene and multiwalled carbon nanotubes and decorated with platinum nanoparticles. Microchim Acta. 2016;183(4):1375-84.

3. Wildi K, Singeisen H, Twerenbold R, Badertscher P, Wussler D, Klinkenberg LJJ, et al. Circadian rhythm of cardiac troponin I and its clinical impact on the diagnostic accuracy for acute myocardial infarction. Int J Cardiol. 2018;(270):14-20.

4. Floriano PN, Christodoulides N, Miller CS, Ebersole JL, Spertus J, Rose BG, et al. Use of Saliva-Based Nano-Biochip Tests for Acute Myocardial Infarction at the Point of Care: A Feasibility Study. Clin Chem. 2009;55(8):1530-8.

5. Zhang B, Morales AW, Peterson R, Tang L, Ye JY. Label-free detection of cardiac troponin I with a photonic crystal biosensor. Biosensors and Bioelectronics. 2014; (58)107-13.

6. Ko S, Kim B, Jo S, Oh SY, Park J. Electrochemical detection of cardiac troponin I using a microchip with the surfacefunctionalized poly(dimethylsiloxane) channel. Biosensors and Bioelectronics. 2007;23(1):51-9.

7. Akter R, Jeong B, Lee Y, Choi J, Rahman MA. Femtomolar detection of cardiac troponin I using a novel label-free and reagentfree dendrimer enhanced impedimetric immunosensor. Biosensors and Bioelectronics. 2017;(91):637-43.

8. Wang J. Electrochemical biosensors: Towards point-of-care cancer diagnostics. Biosensors and Bioelectronics. 2006;21(10):1887-92.

9. Jayanthi VSPK, Das AB, Saxena U. Recent advances in biosensor development for the detection of cancer biomarkers. Biosensors and Bioelectronics. 2017;(91):15-23.

10. Kim J, Stockton AM, Jensen EC, Mathies RA. Pneumatically actuated microvalve circuits for programmable automation of chemical and biochemical analysis. Lab Chip. 2016;16(5):812-9.

11. Thaler M, Luppa PB. Highly sensitive immunodiagnostics at the point of care employing alternative recognition elements and smartphones: hype, trend, or revolution? Anal Bioanal Chem. 2019;411(29):7623-35.

12. Chen C, Zhang X, Zhu J, Li J, Zhang L, Wang E. A nanochannel based on-line universal logic ion sensing platform. Nanoscale. 2013;5(17):8221-6

13. Wang J, Katz E. Digital biosensors with built-in logic for biomedical applications_-biosensors based on a biocomputing concept. Anal Bioanal Chem. 2010;398(4):1591-603.

14. Garcia PT, Guimarães LN, Dias AA, Ulhoa CJ, Coltro WKT. Amperometric detection of salivary $\alpha$-amylase on screen-printed carbon electrodes as a simple and inexpensive alternative for point-of-care testing. Sensors and Actuators B: Chemical. 
2018;(258):342-8.

15. Idili A, Gerson J, Parolo C, Kippin T, Plaxco KW. An electrochemical aptamer-based sensor for the rapid and convenient measurement of 1-tryptophan. Anal Bioanal Chem. 2019;411(19):4629-35.

16. Carvajal S, Fera SN, Jones AL, Baldo TA, Mosa IM, Rusling JF, et al. Disposable inkjet-printed electrochemical platform for detection of clinically relevant HER-2 breast cancer biomarker. Biosensors and Bioelectronics. 2018;(104):158-62.

17. Rezaei B, Shoushtari AM, Rabiee M, Uzun L, Mak WC, Turner APF. An electrochemical immunosensor for cardiac Troponin I using electrospun carboxylated multi-walled carbon nanotube-whiskered nanofibres. Talanta. 2018;(182):178-86.

18. Syedmoradi L, Daneshpour M, Alvandipour M, Gomez FA, Hajghassem H, Omidfar K. Point of care testing: The impact of nanotechnology. Biosensors and Bioelectronics. 2017;(87):373-87.

19. Lu L, Gunasekaran S. Dual-channel ITO-microfluidic electrochemical immunosensor for simultaneous detection of two mycotoxins. Talanta. 2019;(194): 709-16.

20. GUESHI T, TOKUDA K, MATSUDA H. VOLTAMMETRY AT PARTIALLY COVERED ELECTRODES .2. LINEAR POTENTIAL SWEEP AND CYCLIC VOLTAMMETRY. J Electroanal Chem. 1979;101(1):29-38.

21. Davies TJ, Compton RG. The cyclic and linear sweep voltammetry of regular and random arrays of microdisc electrodes: Theory. J Electroanal Chem. 2005;585(1):63-82.

22. Aghamiri ZS, Mohsennia M, Rafiee-Pour H. Immobilization of cytochrome $\mathrm{c}$ and its application as electrochemical biosensors. Talanta. 2018;(176):195-207.

23. Erden PE, Kılıç E. A review of enzymatic uric acid biosensors based on amperometric detection. Talanta. 2013;( 107):312-23. 24. Liu Y, Wang H, Chen J, Liu C, Li W, Kong J, et al. A Sensitive Microchip-Based Immunosensor for Electrochemical Detection of Low-Level Biomarker S100B. Electroanal. 2013;25(4):1050-5.

25. Sassa F, Morimoto K, Satoh W, Suzuki H. Electrochemical techniques for microfluidic applications. Electrophoresis. 2008;29(9):1787-800.

26. Zhang T, Ma N, Ali A, Wei Q, Wu D, Ren X. Electrochemical ultrasensitive detection of cardiac troponin I using covalent organic frameworks for signal amplification. Biosensors and Bioelectronics. 2018;(119):176-81.

27. Mohammadniaei M, Koyappayil A, Sun Y, Min J, Lee M. Gold nanoparticle/MXene for multiple and sensitive detection of oncomiRs based on synergetic signal amplification. Biosensors and Bioelectronics. 2020;(159):112208.

28. Wang H, Zhao X, Yang H, Cao L, Deng W, Tan Y, et al. Three-dimensional macroporous gold electrodes superior to conventional gold disk electrodes in the construction of an electrochemical immunobiosensor for Staphylococcus aureus detection. The Analyst. 2020;145(8):2988-94.

29. Cimafonte M, Fulgione A, Gaglione R, Papaianni M, Capparelli R, Arciello A, et al. Screen Printed Based Impedimetric Immunosensor for Rapid Detection of Escherichia coli in Drinking Water. Sensors-Basel. 2020;20(1):274.

30. Yun S, Lee S, Park JP, Choo J, Lee EK. Modification of phage display technique for improved screening of high-affinity binding peptides. J Biotechnol. 2019;(289):88-92.

31. Green JM. A practical guide to analytical method validation. Anal Chem. 1996;68(9):A305-9.

32. Gustavo González A, Ángeles Herrador M. A practical guide to analytical method validation, including measurement uncertainty and accuracy profiles. TrAC Trends in Analytical Chemistry. 2007;26(3):227-38.

33. Zhu L, Ye J, Yan M, Zhu Q, Wang S, Huang J, et al. Electrochemiluminescence Immunosensor Based on Au Nanocluster and Hybridization Chain Reaction Signal Amplification for Ultrasensitive Detection of Cardiac Troponin I. Acs Sensors. 2019;4(10):2778-85.

34. Singal S, Srivastava AK, Rajesh. Electrochemical Impedance Analysis of Biofunctionalized Conducting Polymer-Modified Graphene-CNTs Nanocomposite for Protein Detection. Nanomicro Lett. 2017;9(1):7.

35. Yola ML, Atar N. Development of cardiac troponin-I biosensor based on boron nitride quantum dots including molecularly imprinted polymer. Biosensors and Bioelectronics. 2019;(126):418-24. 